\title{
Extracellular vesicles-mediated noncoding RNAs transfer in cancer
}

\author{
Pei Ma ${ }^{1 \dagger}$, Yutian Pan ${ }^{1 \dagger}$, Wei $\mathrm{Li}^{1 \dagger}$, Chongqi Sun ${ }^{1}$, Jie Liu', Tongpeng $\mathrm{Xu}^{1}$ and Yongqian Shu ${ }^{1,2^{*}}$
}

\begin{abstract}
Extracellular vesicles (EVs) are small membranous vesicles secreted from numerous cell types and have been found involved in cell-to-cell communication by transferring noncoding RNAs (ncRNAs) including microRNAs, long noncoding RNAs, and circular RNAs. Emerging evidence shows that EV-associated ncRNAs play important roles in a wide range of diseases, particularly in cancer where they function through regulating protein expression of the pivotal genes that make contributions to tumorigenesis. Given their stability and abundance in serum, EV-associated ncRNAs can act as new diagnostic biomarkers and new therapeutic targets for cancer. Herein, we review the properties of EV-associated ncRNAs, their functions, and potential significance in cancer.
\end{abstract}

Keywords: Extracellular vesicles, Noncoding RNAs, Cancer, Mechanism

\section{Background}

Extracellular vesicles (EVs) are small lipid bilayered vesicles released by a wide range of normal or diseased cells. Three main types of EVs are exosomes, microvesicles, and apoptotic bodies which are distinguished on the basis of their size and biogenesis [1,2]. Exosomes are 40 to $100 \mathrm{~nm}$ in diameter and are released by multivesicular bodies. Microvesicles are 50 to $1000 \mathrm{~nm}$ in diameter and are formed by budding directly from the plasma membrane. Apoptotic bodies are 800 to $5000 \mathrm{~nm}$ in diameter and are derived by apoptotic cells [3-5]. The current golden standard method for separating and purifying EVs, differential ultracentrifugation, is incapable of distinguishing between exosomes and microvesicles [6]. In order to avoid unnecessary confusion, we used the term "EVs" in this review.

Noncoding RNAs (ncRNAs) refer to RNAs that cannot be translated into proteins. MicroRNAs (miRNAs) are the most widely studied class of ncRNAs with length of $\sim 22$ nucleotides, which mediate post-transcriptional gene silencing by controlling the translation of mRNA into proteins in animals $[7,8]$. Long noncoding RNAs (lncRNAs) are a

\footnotetext{
*Correspondence: yongqian_shu@163.com; shuyongqian@csco.org.cn Pei Ma, Yutian Pan and Wei Li are joint first author.

${ }^{\dagger}$ Equal contributors

${ }^{1}$ Department of Oncology, the First Affiliated Hospital of Nanjing Medical University, 300 Guangzhou Road, Nanjing 210029, People's Republic of China ${ }^{2}$ Jiangsu Key Lab of Cancer Biomarkers, Prevention and Treatment, Collaborative Innovation Center for Cancer Personalized Medicine, Nanjing Medical University, Nanjing, People's Republic of China
}

heterogeneous group of noncoding transcripts that make up the largest portion of the mammalian noncoding transcriptome with a length of more than 200 nucleotides [9]. LncRNAs are known to regulate gene expression via various mechanisms. For example, they can mediate epigenetic modifications of DNA by recruiting chromatin-remodeling complexes to specific loci $[10,11]$. Other types of ncRNAs include ultraconserved regions (T-UCRs), small nucleolar RNAs (snoRNAs), PIWI-interacting RNAs (piRNAs) as well as circular RNAs (circRNAs), which might also contribute to the development of many different human disorders $[9,12]$. Currently, ncRNAs are found to have diverse biological regulatory functions and dysregulated expression of ncRNAs is closely associated with development of diseases including cancer [12].

EVs that were initially considered as garbage bags for abandoned membrane parcels and molecular fragments were first recognized as being closely related to the function of the immune system with the finding of the role of exosomes in the presentation of B lymphocyte antigens in 1996 by Raposo et al. [13]. In the 2010s, researchers found that miRNA and mRNA can be loaded as "goods" in EVs [14]. In recent years, EVs are discovered to serve as "communication shuttles" between cells and transduct signals between cells [15].

Herein, we will discuss how ncRNAs derived from EVs participate in tumorigenesis, invasion, metastasis, and 
drug resistance and how to use them as new diagnostic biomarkers and therapeutic targets (Fig. 1).

\section{EV-associated miRNAs in cancer}

MiRNAs are a class of small noncoding RNA molecules that can regulate many genes by binding to noncoding regions of target mRNAs, post-transcriptionally lowering mRNAs and proteins [7]. Secreted miRNAs were first identified in human serum and have also been found in several biological fluids including saliva, breast milk, and urine [16]. Recently, as shown by Montecalvo and colleagues, miRNAs transferred by EVs can repress mRNAs in target cells, indicating their role as cell-to-cell communication shuttles and thus having an influence on tumorigenesis and tumor development through various mechanisms [16]. Therefore, EV-associated microRNAs can both promote and suppress tumorigenesis and development, which depends on the function of their target mRNAs and protein product (Table 1) [17].

Clinical features including predict treatment-free survival (TFS) and overall survival (OS) are correlated to the function of EV-associated miRNAs in cancer. For example, miR-150 can be released into the extracellular space via
EVs and it has been shown that among chronic lymphocytic leukemia (CLL) patients, a low cellular miR-150 expression level is associated with tumor burden, disease aggressiveness, and poor prognostic factors while a high level of serum miR-150 is associated with tumor burden markers and some markers of poor prognosis in contrast. Similarly, cellular and serum miR-150 can also influence TFS and OS in an opposite manner: patients with low cellular/serum miR-150 levels have median TFS of 40/ 111 months compared with high-level patients who have a median TFS of $122 / 60$ months $(P<0.0001 / P=0.0066)$. Similar results have been observed for OS [18].

\section{miRNAs transferred by tumor cell-derived EVs in cancer}

Evidence has begun to accumulate that tumor cells have the ability of constitutively secreting a variety of EVs especially containing miRNAs, which has been found to potentially exert a paracrine influence on the surrounding cells to promote proliferation, induce angiogenesis, affect tumor immunity, and result in drug resistance. They can also act on distant organs in an endocrine fashion, which could have profound effect on metastasis $[19,20]$.

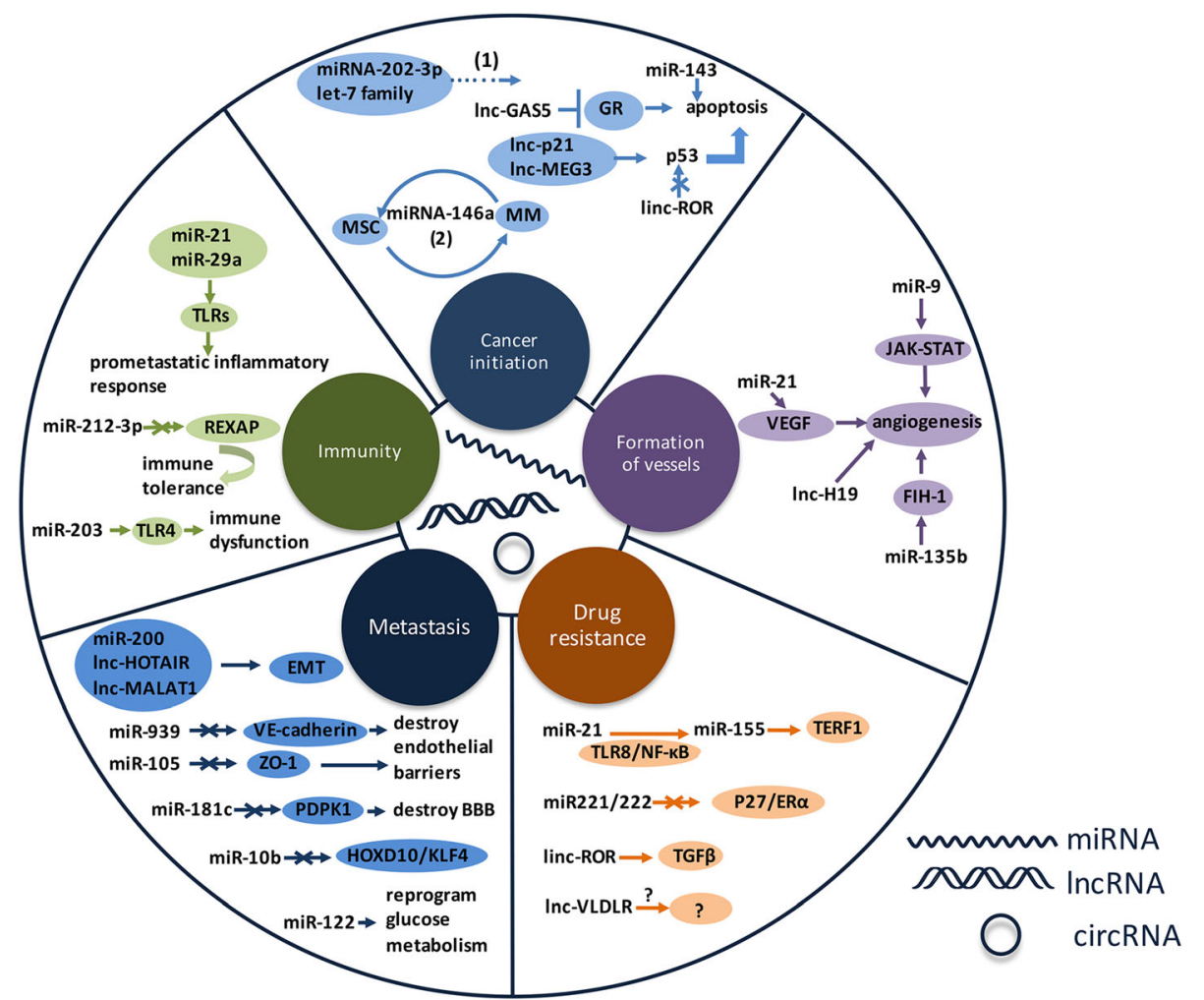

Fig. 1 EV-associated ncRNAs in cancer. EV-associated ncRNAs contribute to the five types of function in cancer. Selected examples of EVassociated ncRNAs and their mechanisms are shown in cancer initiation, formation of vessels, drug resisitance, metastasis, and immunity. (1) Cancerous cells might discard anti-tumorigenic miRNAs via EVs to stimulate cancer initiation and progression. (2) There exists a positive feedback loop between MM cells and MSC that MM cells promote the increase of miR146a in MSC leading to more cytokine secretion, which in turn favors MM cell growth and migration 
Table 1 EV-associated miRNAs in cancer

\begin{tabular}{|c|c|c|c|c|}
\hline miRNA & Cancer type & Biological function & Mechanism & Refs \\
\hline miR-202-3p & CLL & Suppress cancer initiation & Tumors discard these miRNAs via EVs to & {$[27]$} \\
\hline let-7 family & Gastric cancer & Suppress cancer initiation & Promote cancer initiation & [21] \\
\hline miR-146a & MM & Favor MM cell growth & Elevate several cytokines and chemokines & [25] \\
\hline miR-21 & Lung cancer & Promote angiogenesis & Elevate levels of VEGF & [30] \\
\hline $\operatorname{miR}-21$ & Lung cancer & Regulate immunity & Bind as ligands to TLRs in immune cells & [53] \\
\hline miR-21 & Ovarian cancer & Suppress apoptosis & Bind to its target APAF1 & [63] \\
\hline miR-9 & Breast cancer & Promote angiogenesis & Activate JAK-STAT pathway & [36] \\
\hline miR-135b & MM & Promote angiogenesis & Target HIF-1 & [37] \\
\hline miR-939 & Breast cancer & Destroy endothelial barriers & Downregulate VE-cadherin & [38] \\
\hline miR-105 & Breast cancer & Destroy endothelial barriers & Target the tight junction protein ZO-1 & [39] \\
\hline miR-10b & Breast cancer & Promote cell invasion & Suppress its target genes HOXD10 and KLF4 & [40] \\
\hline miR-181c & Breast cancer & Destroy BBB & Downregulate its target gene PDPK1 & [43] \\
\hline miR-200 & Breast cancer & Promote metastasis & Regulate MET process & [47] \\
\hline miR-122 & Breast cancer & Promote metastasis & Reprogram glucose metabolism & [49] \\
\hline miR-29a & Lung cancer & Regulate immunity & Bind as ligands to TLRs in immune cells & [53] \\
\hline miR-203 & PC & Cause immune dysfunction & Regulate TLR4 & [54] \\
\hline miR-212-3p & PC & Induce immune tolerance & Downregulate REXAP expression & [56] \\
\hline miR-221/222 & Breast cancer & Enhance drug resistance & Reduce target gene expression of P27 and ERa & [56] \\
\hline miR-21/155 & Neuroblastoma & Enhance drug resistance & $\begin{array}{l}\text { Function as exosomic miR-21/ TLR8/NF-KB/exosomic } \\
\text { miR-155/TERF1 axis }\end{array}$ & [57] \\
\hline miR-143 & Prostate cancer & Inhibit cell growth & Act as a death signal in cell-competitive process & [62] \\
\hline
\end{tabular}

The first step to study EV-associated miRNAs is isolating EVs from cultured cell lines and validating their quality by analyses of transmission electron microscopy and western blotting. Secondly, RNAs are isolated from cells and culture media, and profiles of miRNA fractions are obtained using microarray analysis. Thirdly, we can observe the abundance of miRNAs in both the intracellular and extracellular fractions by comparing signal intensities of microarray data and the following validation using RT-PCR analysis [21]. We can also study the changes of biological or pathological properties in their target cells and the underlying mechanisms through which they influence cancer development by using MTT and other methods. Finally, nude mouse xenograft models can be used to measure miRNA effects in vivo.

\section{Influence on cancer initiation}

Tumorigenesis is attributed to a two-way interaction between cancer cells and the surrounding microenvironment rather than a tumor cell-autonomous mechanism triggered by accumulation of somatic aberrations [22].

Mesenchymal stem cells (MSCs), defined as multipotent stem cells that have the capacity to give rise to adipocytes, osteoblasts, and chondrocytes, are an important component of the tumor microenvironment [23]. Tumor cells can reprogram surrounding MSCs into tumor supportive myofibroblasts through intercellular communications, especially by releasing EVs. This long-time "education" by tumor cells contributes to the cancer initiation [24]. MiRNAs transferred by cancerous cell-derived EVs can promote cancer initiation. A positive feedback loop between multiple myeloma (MM) cells and MSCs is that MM cells promote the increase of miR146a in MSCs, leading to more cytokine secretion, which in turn favors MM cell growth and migration. MM cells secrete EVs containing miR-146a into MSC and the overexpression of miR-146a in MSC elevates secretion of several cytokines and chemokines including CXCL1, IL-6, IL-8, IP-10, MCP-1, and CCL-5, enhancing MM cell viability and migration as a result, which expands our knowledge of mutual communication between cells mediated by EV-associated miRNAs [25].

On the other hand, cancerous cells might discard antitumorigenic miRNAs via EVs to stimulate cancer initiation and progression [26]. For example, exosomal release of miR-202-3p from CLL cells into the microenvironment increases the expression of its target "suppressor of fused" (Sufu), a negative regulator of Hedgehog signaling, resulting in a decrease of its anti-tumorigenic effect [27]. Similarly, a metastatic gastric cancer cell line, AZ-P7a cells selectively secrete let-7 family miRNAs, which are considered mainly as tumor suppressor genes targeting oncogenes such as RAS and high-mobility group A2 (HMGA2), into the 
extracellular environment via exosomes to maintain their tumorigenic and metastatic propensities [21]. These results provide the basis for the hypothesis that cancerous cells specifically package tumor-suppressive miRNAs into exosomes to promote cancer initiation.

\section{Involvement in tumor angiogenesis}

Angiogenesis refers to the formation of tumorassociated vessels, which is the result of an interplay between cancer cells and endothelial cells and results in the sprouting of locally pre-existing vessels or the recruitment of bone marrow-derived endothelial progenitor cells [26]. Recent studies have highlighted the functions of EV-associated miRNAs on angiogenesis and tumor development.

Vascular endothelial growth factor (VEGF) functions as an important factor in angiogenesis as they can bind to receptors to induce endothelial cell migration and form new blood vessels whose elevation significantly fosters angiogenesis and tumor development [28, 29]. MiR-21 in exosomes derived from transformed human bronchial epithelial (HBE) cells elevates levels of VEGF in HBE cells by activating STAT3, which promotes angiogenesis and malignant transformation of HBE cells [30].

Furthermore, miR-9 is a new star in regulating tumor angiogenesis by modulating the JAK-STAT pathway in endothelial cells. STAT proteins not only play a crucial role in tumor cell proliferation, survival, and invasion but also significantly contribute to the formation of a unique tumor microenvironment [31, 32]. Emerging evidence has shown that there exists a link between STATs activation in endothelial cells and tumor angiogenesis [33-35]. Exogenous miR-9 effectively reduces SOCS5 levels, leading to activated JAK-STAT pathway, which promotes endothelial cell migration and tumor neovascularization [36].

MiR-135b has also been found to be transferred into endothelial cells through exosomes by MM cells and target a factor-inhibiting hypoxia-inducible factor 1 (HIF1), thus enhancing angiogenesis [37].

\section{Promotion of tumor metastasis}

Besides influencing located cells, cancer-derived EVs can affect cells in distant tissues and organs via delivering miRNAs.

Intravasation, the first step of metastasis cascade, allows the invasion of cancer cells through the basal membrane followed by local infiltration of the stroma-rich extracellular matrix (ECM), potentially leading to metastasis [26]. Modica and colleagues demonstrate an extracellular pro-tumorigenic role for tumor-derived, exosome-associated miR-939 that leads to an increase of monolayer permeability by targeting VE-cadherin and disrupting the endothelial barrier [38].
Additionally, exosome-mediated transfer of cancersecreted miR-105 regulates tumor migration through targeting the tight junction protein $\mathrm{ZO}-1$ and destroys these natural barriers against metastasis. This explains the phenomenon that overexpression of miR-105 in nonmetastatic cancer cells induces metastasis, whereas inhibition of miR-105 in highly metastatic tumors alleviates these effects [39].

Given that cell invasion is a key process in tumor metastasis, exosome-mediated miR-10b secretion is dramatically higher in metastatic breast cancer MDAMB-231 cells than in nonmetastatic breast cancer cells or nonmalignant breast cells. MiR-10b suppresses the protein level of its target genes such as HOXD10 and KLF4, indicating their functional significance [40].

Brain metastasis leads to a particularly poor prognosis for cancer patients [41], with accumulating evidence suggesting that the destruction of the blood-brain barrier (BBB) is one of the key features of brain metastasis [42]. EVs can trigger the breakdown of BBB via delivery of miRNAs. For example, EVs containing miR-181c promote the destruction of $\mathrm{BBB}$ via degradation of its target gene, PDPK1, which leads to the downregulation of phosphorylated cofilin and the resultant-activated cofilin-induced modulation of actin dynamics [43].

Epithelial to mesenchymal transition (EMT) is reversible and the reciprocal mesenchymal to epithelial transition (MET) process allows cancerous cells to regain epithelial properties and integrate into distant organs, thus promoting long-distance metastasis $[44,45]$. Members of the miR-200 family (miR-200a, miR-200b, miR-200c, miR-429, miR-141), which are enriched in the serum of patients with metastatic cancers, share the same seed sequence and the same targets. They have the ability of regulating MET process in large part by inhibiting the expression of Zeb1 and Zeb2, the transcriptional repressors of many epithelial genes [46]. Upon integrated into nonmetastatic cells, miR-200 microRNAs in EVs derived from metastatic cells alter gene expression and transfer metastatic capability [47].

Reprogrammed energy metabolism to fuel rapid cell growth and proliferation is an emerging hallmark of cancer [47]. It has been shown by Fong et.al that cancer cells are able to suppress glucose uptake by nontumor cells in the pre-metastatic niche, by secreting EVs abundant in miR-122. High miR-122 levels in the circulation are associated with metastatic progression in BC patients [48] and cancer cell-secreted miR-122 facilitates metastasis by increasing nutrient availability in the pre-metastatic niche [49]. MiR-122 suppresses glucose uptake by niche cells in vitro and in vivo by downregulating the glycolytic enzyme pyruvate kinase (PKM) [49]. 
Above all, EV-associated miRNAs can promote tumor long-distance metastasis through a wide range of mechanisms.

\section{Regulation of tumor immunity}

The topic that miRNAs transferred by exosomes can regulate tumor immunity has now blossomed into a fullfledged field of research.

Dendritic cells (DCs) are typical antigen-presenting cells (APCs) which express a wide range of toll-like receptors (TLRs) and cytokines, playing an important role in activation of immune response [50]. TLRs lead to cell activation and cytokine production by recognizing and binding viral single-stranded RNA sequences on dendritic cells and B lymphocytes [51, 52].

Tumor-secreted miR-21 and miR-29a trigger a TLRmediated prometastatic inflammatory response by binding as ligands to receptors of TLR family, namely murine TLR7 and human TLR8, in immune cells. This prompts the very first attempt at studying EV-associated miRNAs as paracrine agonists of TLRs and key regulators of the tumor microenvironment, suggesting their involvement in tumor-immune system communication and importance in tumor growth and spread [53].

Evidence has demonstrated that miR-203 may cause immune dysfunction as they can be transferred via exosomes to interfere with DCs and contribute to dysfunction of DCs by acting as the regulator of TLR4 and production of cytokines such as TNF-a and IL-12 [54]. In other circumstances, pancreatic cancer (PC)-derived exosomal miRNAs can inhibit mRNA expression of DCs and induce immune tolerance. Regulatory factor X-associated protein (RFXAP) is a key transcription factor for the MHC II gene whose deficiency can lead to a rare severe immunodeficiency disorder termed bare lymphocyte syndrome. MiR-212-3p transferred from $\mathrm{PC}$-secreted exosomes downregulate RFXAP expression, inhibiting MHC class II expression and leading to inactivation of CD4+ T-lymphocytes [55].

\section{Contribution to drug resistance}

Drug resistance represents a daunting challenge to the successful treatment of all kinds of cancers. Results from Yifang Wei are the first to show that secreted miR-221/ 222 acts as signaling molecules to mediate communication of tamoxifen resistance. MCF-7TamR exosomes enter into MCF-7wt cells where they release miR-221/ 222 , and the elevated miR-221/222 effectively reduce the target gene expression of P27 and ERa, enhancing tamoxifen resistance in recipient cells [56].

Challagundla and colleagues have also identified that neuroblastoma cells secrete exosomic miR-21, leading to a TLR8 and NF-кB-dependent upregulation of miR-155. Exosomic miR-155 transferred by human monocytes was capable of directly targeting TERF1 and affecting telomerase activity and telomere length in NBL, which is involved in the increased chemoresistance CDDP. This novel exosomic miR-21/ TLR8/NF-kB/exosomic miR$155 /$ TERF1 axis suggests that exosomes within the tumor microenvironment are important molecular targets to restore drug sensitivity [57].

While tumor-derived EVs modify the function of immune cells, immune cell-derived EVs can be used to treat cancer cells. An example is that DCs secrete EVs expressing functional major histocompatibility complex class I and class II, and $T$ cell costimulatory molecules as antigen-presenting vesicles. Tumor peptide-pulsed DCderived exosomes target specific cytotoxic T-lymphocytes in vivo and eradicate growth of established murine tumors in a $\mathrm{T}$ cell-dependent fashion, which can be utilized as cell-free vaccines for suppressing tumor growth [58].

\section{miRNAs transferred by noncancerous cell-derived EVs in cancer}

We need to understand that tumors consist not only of malignant cells but also of a variety of stromal cell types including three subtypes: angiogenic vascular cells, infiltrating immune cells, and cancer-associated fibroblastic cells as well as ECM. MiRNAs in stromal cell-derived EVs make contributions to resisting cell death, avoiding immune destruction, activating invasion, inducing angiogenesis, and sustaining proliferative signaling [44, 59].

There exists a homeostatic cell-competitive system where normal epithelial cells secrete tumor-suppressive miRNAs via EVs to prevent the aberrant growth of neighboring cells. The failure of this system is considered to be a reason for tumor initiation [60-62]. Of these miRNAs, miR-143 has been shown to act as a death signal in the cell-competitive process and induce growth inhibition exclusively in prostate cancer cells in vitro and in vivo, which provides a novel insight into a tumor initiation mechanism [62].

MiR-21, a famous and well-studied microRNA, has been revealed to play a pivotal role in cancer proliferation, angiogenesis [30], tumor immunity [53], and drug resistance [57] as discussed above. Transferred from CAFs to the cancer cells, miR-21 suppresses ovarian cancer apoptosis and confers chemoresistance by binding to its direct novel target, APAF1. This leads to very open and constructive discussion that miR-21 delivered by exosomes derived from neighboring stromal cells in the omental tumor microenvironment can alter the malignant phenotype of metastatic ovarian cancer cells, indicating their potential function in tumor therapy [63].

\section{EV-associated IncRNAs in cancer IncRNAs secreted by EVs}

Long noncoding RNAs (lncRNAs) are RNA transcripts greater than 200 nucleotides in length, and they play 
important regulatory roles in gene expression [64, 65]. Once considered little more than genomic noise, recent observations have proved that lncRNAs can act as miRNA sponges [66] and mediate normal cellular processes through various mechanisms such as epigenetic regulation, chromatin remodeling, and transcriptional or post-transcriptional regulation or modulation of protein function and localization [67].

Several previously described lncRNAs such as MALAT1, HOTAIR, and GAS5 have been discovered to be expressed at higher levels within exosomes from HeLa and MCF-7 cells, suggesting that lncRNAs and exosomes may function together to disseminate cell signals that alter local cellular microenvironment and result in a phenotypic effect within the recipient cells (Table 2) [68].

\section{Functions of EV-associated IncRNAs in cancer}

LncRNAs can influence regulation of gene expression and have an impact on many different cancerous processes, where they can influence tumorigenesis, invasion, and metastasis.

The study of EV-associated lncRNAs begins with collecting tumor tissues and matched adjacent nontumor tissues. Then, lncRNA exposure levels are determined using RT-PCR and in situ hybridization and the exposure levels of the proteins are measured by western blot. In addition, dual-luciferase report assay can be performed to verify the target effect of IncRNAs on miRNAs. The proliferation, invasion, and migration ability of target cells after being infected were tested by MTT assay, wound healing assay, and transwell assays. Finally, researchers can utilize nude mouse xenograft models to measure lncRNA effects on tumor growth in vivo [69].

LncRNA-p21 and lncRNA-GAS5 are tumor suppressor molecules in the cellular machinery [70, 71].
Significantly higher level of exosomal lncRNA-p21 is observed in the patients with prostate cancer, which is stimulated by the p53 tumor suppressor protein. Upon transcription, it can suppress the expression of the genes transcriptionally regulated by $\mathrm{p} 53$ by binding to the hnRNP-K complex [71]. LncRNA-GAS5 secreted by prostate cancer cells is an important mediator of inducing apoptosis since it suppresses several antiapoptotic genes by binding to the DNA-binding domain of the glucocorticoid receptor (GR), which prevents glucocorticoid response elements (GRE) from binding to the GR [72]. The lncRNA MEG3 is an example of another lncRNA that acts as a tumor suppressor gene. MEG3 functions by stimulating p53 expression and can also inhibit cell proliferation independent of the p53 protein [73].

EV-associated lncRNAs can foster tumor development through diverse mechanisms. Accumulating evidence has demonstrated that IncRNA-HOTAIR has the capability of facilitating tumor initiation and progression and is associated with poor prognosis in several cancers [74]. Importantly, lncRNA-HOTAIR functions by regulating several genes involved in EMT including snail family zinc finger 1 (SNAI1), laminin, beta 3 (LAMB3); laminin, gamma 2 (LAMC2); junctional adhesion molecule 2 (JAM2); and ABL proto-oncogene 2 (ABL2) [11, 75-77], as well as participating in the silencing of miR-205 expression in UBC cells through epigenetic regulation. MiR-205 targets the cell-cycle regulation gene cyclin $\mathrm{J}(\mathrm{CCNJ})$ and is proved relevant to the inhibition of proliferation, migration, and invasion of the urothelial bladder cancer cell lines [78].

LncRNA-MALAT1 is also involved in modulating EMT through regulating the expression of proteins concerning EMT, including E-cadherin, ZO-1, bcatenin, vimentin, and snail [79]. Another study indicates that IncRNA-MALAT1 promotes proliferation

Table 2 EV-associated IncRNAs in cancers

\begin{tabular}{lllll}
\hline IncRNA & Cancer type & Biological function & Mechanism & Refs \\
\hline IncRNA-p21 & Prostate cancer & Suppress cancer initiation & $\begin{array}{l}\text { Enhance drug resistance; suppress the genes } \\
\text { regulated by p53 }\end{array}$ & [71] \\
GAS5 & Prostate cancer & Suppress cancer initiation & Suppress several anti-apoptotic genes \\
MEG3 & Lung cancer & Suppress cancer initiation & Stimulate p53 expression & [72] \\
HOTAIR & Bladder cancer & Facilitate tumor progression & Regulate EMT and act as miR-205 sponge \\
MALAT1 & Cervical cancer & Facilitate tumor progression & Modulate EMT & [73] \\
MONC & AKML & Facilitate tumor progression & Act as miR-99a/100 125b sponge \\
MIR100HG & AKML & Facilitate tumor progression & Same as Inc-MONC \\
H19 & Liver cancer & Promote angiogenesis & Affect phenotype of endothelial cells \\
linc-ROR & HCC & Promote tumor progression & Inhibit p53 and act as miR-145 sponge \\
linc-ROR & HCC & Enhance drug resistance & Elevate TGF level & [79] \\
Linc-VLDLR & HCC & Enhance drug resistance & Unknown \\
InCARSR & Renal cancer & Enhance drug resistance & Act as a ceRNA for miR-34 and miR-449 \\
\hline
\end{tabular}


and invasion in cervical cancer cells by Hela and CaSki [80].

LincRNAs MONC and MIR100HG are mainly localized in the nucleus and highly expressed in acute megakaryoblastic leukemia (AMKL) blasts, whose expression is correlated with corresponding miR-99a/100 125b clusters, resulting in tumorigenesis and tumor development. It has been shown that MONC or MIR100HG knockdown inhibits leukemic growth of AMKL cell lines and cells from primary patient samples through a shRNA-induced loss-of-function study [81].

CD90 is a $25-37 \mathrm{kDa}$ glycophosphatidylinositolanchored protein involved in cell-to-cell and cell-matrix interaction, apoptosis, adhesion, migration, fibrosis, and cancer development [U]. Concerning the liver, CD90 is expressed by hepatic stem/progenitor cells [V] and is correlated with an aggressive phenotype during tumor growth, low differentiated HCC, and poor prognosis [82-85]. LncRNA H19, enriched in exosomes released by CD90+ cancer cells rather than parental hepatoma cells, plays an important role in the exosome-mediated phenotype of endothelial cells, thus promoting angiogenesis and cell-to-cell adhesion [86].

Linc-RoR is enriched in tumor cell-derived EVs during hypoxia and can modulate cellular signaling and cell survival in recipient cells as a stress-responsive lncRNA [87]. Linc-RoR prevents the activation of cellular stress pathways, such as the p53 response, promoting survival of iPSCs, and embryonic stem cells [88]. Linc-RoR has been shown to function as a miRNA sponge to miR-145 and modulate the expression of key effectors of the hypoxia response, such as HIF- $1 \alpha$ expression, which contributes to the acute hypoxic response and can promote the expression of several hypoxia-inducible genes associated with angiogenesis, cell growth, differentiation, survival, and apoptosis [89]. Recent studies have identified a previously unrecognized role of linc-ROR as a mediator of cell-to-cell communication through the transfer of extracellular vesicles, resulting in acquired chemoresistance within tissues. EV-associated linc-ROR elevate transforming growth factor (TGF) level in recipient cells, which contributes to loss of therapeutic effect of agents such as sorafenib [90].

Another example of lncRNAs that enhance chemoresistance is linc-VLDLR. Takahashi and colleagues [67] have proposed the potential role of linc-VLDLR as a novel signaling mediator that can contribute to chemotherapeutic stress responses in hepatocellular cancer (HCC) through extracellular vesicle-mediated intercellular signaling and justified more studies to define the mechanisms, which are currently unknown. Besides, lncARSR has been found to be involved in receptor tyrosine kinase inhibitor sunitinib resistance in renal cancer [91]. Localized in the cytoplasm, lncARSR whose expression is correlated with clinical poor sunitinib response might function as a competing endogenous RNA (ceRNA) to sequester miRNAs, resulting in the liberation of corresponding miRNAtargeted transcripts $[92,93]$. LncARSR could be packaged into exosomes and transferred to recipient cells to promote sunitinib resistance via competitively sponging miR$34 / 449$ to promote AXL and c-MET expression in renal cancer cells [68].

\section{EV-associated circRNAs in cancer}

CircRNAs are a class of novel endogenous ncRNAs that form a covalently closed continuous loop unlike linear ncRNAs [94] and provide new insights into the study of ncRNAs because of their tissue and developmental stage-specific expression [95]. Recently, circRNAs have been shown to function as miRNA sponges and RNAbinding protein (RBP) sequestering agents as well as transcriptional regulators to influence gene expression, which sets the foundation of the hypothesis that circRNAs transferred by EVs can take part in cell-to-cell communication with recipient cells [95, 96]. According to the research by Yan Li, abundant circRNAs are contained in EVs compared to the producer cells and changes of associated miRNA levels in producer cells may manipulate the sorting of circRNAs into EVs. For example, EV-associated circRNAs have been found to retain biological activity as growth suppressor by abrogating miR-7 [97]. The study of the mechanisms by which EV-associated circRNAs function in the process of cancer is still on the way. Hopefully, it will represent a research hotspot in the field of EV-associated ncRNAs.

\section{The future of EV-associated ncRNAs}

As described above, the rapid development of EVassociated ncRNAs has contributed to reveal underlying mechanisms of cancer initiation and progression. However, there remain a large number of challenges.

The first issue is that since the current method is incapable of distinguishing between exosomes and microvesicles [6], it is controversial whether the function of EVs is related only to the ncRNAs that is encapsulated in them but not to its form. Therefore, more advanced technology should be used for separating and purifying each subtype of EVs.

Secondly, we should pay special attention to the tissue type being studied. The ncRNAs might serve as a tumor suppressor in one cell type and an oncogene in another. For example, in general, let-7 miRNAs act as a tumor suppressor by targeting oncogenes such as RAS and HMGA2 and let-7 miRNAs are downregulated in many cancers from solid organs [98], while they act as oncogenes since a metastatic gastric cancer cell line named AZ-P7a releases let-7 miRNAs into the extracellular environment to maintain their oncogenesis and 
invasiveness [21]. Therefore, it is important and necessary to clarify what tissue type AZ-P7a cells represent in the research.

The third issue is lacking of a universal method to analyze EV-associated ncRNAs which often leads to discrepancies between studies performed by different groups. Besides, occurrence of EMV-mediated ncRNA transfer has been indirectly confirmed by detecting the altered expression levels of internal miRNAs in both donor and recipient cells. In the future, fluorescence signal amplification by a confocal imaging system may open new avenues for studying EMV transfer directly [99].

EVs are secreted by most cell types and exist in various body fluids including the blood, urine, and saliva. NcRNAs contained in EVs represented the biological or pathological states of cells. Because of their resistance to endogenous RNase and high stability under different storage conditions, EV-associated ncRNAs can serve as valuable noninvasive biomarkers for the diagnosis and prognosis of cancers including glioblastoma melanoma, liver cancer, gastric cancer, ovarian cancer, breast cancer, lung carcinoma, and so on (Table 3) [100-107]. Importantly, panels consisting of a collection of several ncRNAs rather than single miRNA, lncRNA, or circRNA will be necessary to precisely evaluate the diagnosis and prognosis of any kind of cancer [74].

EV-associated ncRNAs have also provided new opportunities for the treatment of cancer. EVs are in small size and capable of crossing biological membranes and protect their cargo from degradation, which suggests that they are ideal delivery systems for the transfer of specific molecules such as miRNAs or anti-miRNAs [108].
For example, it has been discovered that miR-122transfected adipose tissue-derived MSC (AMSC) can effectively package miR-122 into secreted exosomes rendering cancer cells sensitive to chemotherapeutic agents through alteration of miR-122-target gene expression in HCC cells by mediating miR-122 communication between AMSCs and HCC cells. Furthermore, intra-tumor injection of 122-Exo significantly increased the antitumor efficacy of sorafenib on HCC in vivo which suggests that the export of miR-122 via AMSC exosomes represents a novel strategy to enhance HCC chemosensitivity and can be used for HCC therapy [109].

Recently, microvesicles have also been used as the shuttle of delivering antisense RNA targeted to miR-150 that is proved as an oncomir by regulating the VEGF secretion of TAMs into mice to treat tumors, leading to a new avenue for the transfer of antineoplastic drugs [110].

However, the therapeutic potential of EV-associated ncRNAs in cancer is largely unknown. The intracellular delivery of EV-associated proteins can be fulfilled by a new tool named "exosomes for protein loading via optically reversible protein-protein interactions" (EXPLORs) [111]. Similar tools may be also utilized in the study of EV-associated ncRNAs and further research is in urgent need before used in clinic.

\section{Conclusions}

EVs, previously largely thought to function like garbage bags to remove excess or unnecessary constituents from the cells, have now been discovered to be mediators in specific cell-to-cell communication via transferring

Table 3 Current available EV-associated ncRNAs as tumor biomarkers

\begin{tabular}{llll}
\hline EV-associated ncRNA & Cancer type & Biological function & Refs \\
\hline miR-223 & Breast cancer & Promote invasion & [100] \\
let-7 family & Gastric cancer & Suppress cancer initiation & [21] \\
miR-17-92 & Leukemia & Enhance migration and tube formation & [101] \\
miR-15 & MM & Facilitate progression & [102] \\
miR-125b & Melanoma & Monitor indicator & [103] \\
miR-16 & HCC & Mediate intercellular communication \\
miR-21 & Breast cancer & Monitor indicator & [104] \\
miR-21/155 & Neuroblastoma & Enhance drug resistance & [108] \\
H19 & Liver cancer & Promote angiogenesis & [57] \\
HOTAIR & Bladder cancer & Facilitate tumor progression & [86] \\
MALLATI & Cervical cancer & Facilitate tumor progression & [78] \\
MEG3 & Lung cancer & Suppress cancer initiation & [79] \\
GAS5 & Prostate cancer & Suppress cancer initiation & [72] \\
lincRNA-ROR & HCC & Promote tumor progression & [88, 89] \\
Inc-ATB & HCC & Promote metastasis & [106] \\
Inc-PVT1 & HCC & Promote cell proliferation & \\
\hline
\end{tabular}


proteins, RNAs and DNAs. Exosomes are the most widely studied EVs. They can transfer information to the target cells through three main ways: receptor-ligand interaction, direct fusion with plasma membrane, and endocytosis by phagocytosis [112]. Amongst the components they transfer, ncRNAs have drawn the great interest of researchers since emerging evidence has suggested that EV-associated ncRNAs fulfill important functions in the regulation of gene expression and participate in the process of versatile diseases, particularly in cancer. NcRNAs derived from both cancerous cells and noncancerous cells influence on tumorigenesis, angiogenesis, metastasis, immunity, and drug resistance through diverse mechanisms. However, an outstanding question remains as to whether EV-associated ncRNAs actually function in vivo and more research utilizing convenient in vivo model systems are needed as a result. Further studies will likely also focus on the potential role of EVassociated ncRNAs as hopeful diagnostic biomarkers and novel treatment modalities, which will contribute to the health of human beings.

\begin{abstract}
Abbreviations
ABL2: ABL proto-oncogene 2; APCs: Antigen-presenting cells; BBB: Bloodbrain barrier; CCNJ: Cell-cycle regulation gene cyclin J; circRNAs: Circular RNAs; CLL: Chronic lymphocytic leukemia; DCs: Dendritic cells; ECM: Extracellular matrix; EMT: Epithelial to mesenchymal transition; EVs: Extracellular vesicles; FlH: Factor-inhibiting hypoxia-inducible; GAS5: Growth arrest-specific 5; GR: Glucocorticoid receptor; GRE: Glucocorticoid response elements; HBE: Human bronchial epithelial; HCC: Hepatocellular cancer; HMGA2: High-mobility group A2; HOTAIR: HOX antisense intergenic RNA; JAM2: Junctional adhesion molecule 2; LAMB3: Laminin, beta 3; LAMC2: Laminin, gamma 2; Linc-RoR: Long intergenic nonprotein coding RNA, regulator of reprogramming; lincVLDLR: The large intergenic noncoding RNA-VLDLR; InCARSR: InCRNA activated in RCC with sunitinib resistance; IncRNAs: Long noncoding RNAs; MALAT1: Metastasis-associated lung adenocarcinoma transcript 1 ; MET: Mesenchymal to epithelial transition; miRNAs: MicroRNAs; MM: Multiple myeloma; MSC: Mesenchymal stromal cells; ncRNAs: Noncoding RNAs; PC: Pancreatic cancer; piRNAs: Piwi-interacting RNAs; RBP: RNA-binding protein; RFXAP: Regulatory factor X-associated protein; SNAl1: Snail family zinc finger 1; Sufu: Suppressor of fused; TGF: Transforming growth factor; TLRs: Toll-like receptors; VEGF: Vascular endothelial growth factor.
\end{abstract}

\section{Acknowledgements}

Not applicable.

\section{Funding}

This work was supported by grants from the National Natural Science Foundation of China (No. 81672896; NO. 81272532), the Jiangsu Province Clinical Science and Technology Projects (Clinical Research Center, BL2012008), and the Priority Academic Program Development of Jiangsu Higher Education Institutions (JX10231801).

\section{Availability of data and materials}

The material supporting the conclusion of this review has been included within the article.

\section{Authors' contributions}

YS and PM designed the research. YP and PM drafted the manuscript. WL critically revised the manuscript. CS, JL, and TX discussed and revised the manuscript. All authors read and approved final manuscript.

\section{Competing interests}

The authors declare that they have no competing interests.
Consent for publication

Not applicable.

Ethics approval and consent to participate

Not applicable.

Received: 25 November 2016 Accepted: 21 February 2017

Published online: 23 February 2017

\section{References}

1. Kagiya T. MicroRNAs and osteolytic bone metastasis: the roles of microRNAs in tumor-induced osteoclast differentiation. J Clin Med. 2015;4(9):1741-52.

2. Mohankumar S, Patel T. Extracellular vesicle long noncoding RNA as potential biomarkers of liver cancer. Brief Funct Genomic. 2016:15(3):249-56.

3. Carandini T, Colombo F, Finardi A, Casella G, Garzetti L, Verderio C, Furlan R. Microvesicles: what is the role in multiple sclerosis? Front Neurol. 2015;6:111.

4. Nishida-Aoki N, Ochiya T. Interactions between cancer cells and normal cells via miRNAs in extracellular vesicles. Cell Mol Life Sci. 2015;72(10):1849-61.

5. Crescitelli R, Lasser C, Szabo TG, Kittel A, Eldh M, Dianzani I, Buzas El, Lotvall J. Distinct RNA profiles in subpopulations of extracellular vesicles: apoptotic bodies, microvesicles and exosomes. J Extracellul Vesicles. 2013;2:20677.

6. Thery C, Amigorena S, Raposo G, Clayton A. Isolation and characterization of exosomes from cell culture supernatants and biological fluids. Curr Protoc Cell Biol. 2006; Chapter 3:Unit 322.

7. He L, Hannon GJ. MicroRNAs: small RNAs with a big role in gene regulation. Nat Rev Genet. 2004;5(7):522-31.

8. Mendell JT. MicroRNAs: critical regulators of development, cellular physiology and malignancy. Cell Cycle. 2005;4(9):1179-84.

9. Mercer TR, Dinger ME, Mattick JS. Long non-coding RNAs: insights into functions. Nat Rev Genet. 2009;10(3):155-9.

10. Navarro P, Page DR, Avner P, Rougeulle C. Tsix-mediated epigenetic switch of a CTCF-flanked region of the Xist promoter determines the Xist transcription program. Genes Dev. 2006;20(20):2787-92.

11. Gupta RA, Shah N, Wang KC, Kim J, Horlings HM, Wong DJ, Tsai MC, Hung T, Argani P, Rinn JL, et al. Long non-coding RNA HOTAIR reprograms chromatin state to promote cancer metastasis. Nature. 2010;464(7291):1071-6.

12. Mattick JS, Makunin IV. Non-coding RNA. Hum Mol Genet. 2006;15 Spec No 1:R17-29.

13. Raposo G, Nijman HW, Stoorvogel W, Liejendekker R, Harding CV, Melief CJ, Geuze HJ. B lymphocytes secrete antigen-presenting vesicles. J Exp Med. 1996;183(3):1161-72

14. Braicu C, Tomuleasa C, Monroig P, Cucuianu A, Berindan-Neagoe I, Calin GA Exosomes as divine messengers: are they the Hermes of modern molecular oncology? Cell Death Differ. 2015;22(1):34-45.

15. Tkach $\mathrm{M}$, Thery C. Communication by extracellular vesicles: where we are and where we need to go. Cell. 2016;164(6):1226-32.

16. Montecalvo A, Larregina AT, Shufesky WJ, Stolz DB, Sullivan ML, Karlsson JM, Baty CJ, Gibson GA, Erdos G, Wang Z, et al. Mechanism of transfer of functional microRNAs between mouse dendritic cells via exosomes. Blood. 2012;119(3):756-66.

17. Bronisz A, Godlewski J, Chiocca EA. Extracellular vesicles and microRNAs: their role in tumorigenicity and therapy for brain tumors. Cell Mol Neurobiol. 2016;36(3):361-76.

18. Stamatopoulos B, Van Damme M, Crompot E, Dessars B, Housni HE, Mineur $P$, Meuleman N, Bron D, Lagneaux L. Opposite prognostic significance of cellular and serum circulating microRNA-150 in patients with chronic lymphocytic leukemia. Mol Med. 2015;21:123-33.

19. Chen $X$, Liang $H$, Zhang J, Zen $K$, Zhang CY. Secreted microRNAs: a new form of intercellular communication. Trends Cell Biol. 2012;22(3):125-32.

20. Azmi AS, Bao B, Sarkar FH. Exosomes in cancer development, metastasis, and drug resistance: a comprehensive review. Cancer Metastasis Rev. 2013; 32(3-4):623-42.

21. Ohshima K, Inoue K, Fujiwara A, Hatakeyama K, Kanto K, Watanabe Y, Muramatsu K, Fukuda Y, Ogura S, Yamaguchi K, et al. Let-7 microRNA family is selectively secreted into the extracellular environment via exosomes in a metastatic gastric cancer cell line. PLoS One. 2010;5(10):e13247.

22. Baroni S, Romero-Cordoba S, Plantamura I, Dugo M, D'Ippolito E, Cataldo A, Cosentino G, Angeloni V, Rossini A, Daidone MG, et al. Exosome-mediated delivery of induces cancer-associated fibroblast-like properties in human breast fibroblasts. Cell Death Dis. 2016;7(7):e2312. 
23. Pittenger MF, Mackay AM, Beck SC, Jaiswal RK, Douglas R, Mosca JD, Moorman MA, Simonetti DW, Craig S, Marshak DR. Multilineage potential of adult human mesenchymal stem cells. Science. 1999;284(5411):143-7.

24. Li X, Wang S, Zhu R, Li H, Han Q, Zhao RC. Lung tumor exosomes induce a pro-inflammatory phenotype in mesenchymal stem cells via NFkappaB-TLR signaling pathway. J Hematol Oncol. 2016;9:42

25. De Veirman K, Wang J, Xu S, Leleu X, Himpe E, Maes K, De Bruyne E, Van Valckenborgh E, Vanderkerken K, Menu $E$, et al. Induction of miR-146a by multiple myeloma cells in mesenchymal stromal cells stimulates their protumoral activity. Cancer Lett. 2016;377(1):17-24.

26. Dhondt B, Rousseau Q, De Wever O, Hendrix A. Function of extracellular vesicle-associated miRNAs in metastasis. Cell Tissue Res. 2016;365(3):621-41.

27. Farahani M, Rubbi C, Liu L, Slupsky JR, Kalakonda N. CLL exosomes modulate the transcriptome and behaviour of recipient stromal cells and are selectively enriched in miR-202-3p. PLoS One. 2015;10(10):e0141429.

28. Ferrara N, Gerber HP, LeCouter J. The biology of VEGF and its receptors. Nat Med. 2003;9(6):669-76

29. Bolat F, Kayaselcuk F, Nursal TZ, Yagmurdur MC, Bal N, Demirhan B. Microvessel density, VEGF expression, and tumor-associated macrophages in breast tumors: correlations with prognostic parameters. J Exp Clin Cancer Res. 2006;25(3):365-72

30. Liu Y, Luo F, Wang B, Li H, Xu Y, Liu X, Shi L, Lu X, Xu W, Lu L, et al. STAT3regulated exosomal miR-21 promotes angiogenesis and is involved in neoplastic processes of transformed human bronchial epithelial cells. Cancer Lett. 2016;370(1):125-35.

31. Lee H, Herrmann A, Deng JH, Kujawski M, Niu G, Li Z, Forman S, Jove R, Pardoll DM, Yu H. Persistently activated Stat3 maintains constitutive NFkappaB activity in tumors. Cancer Cell. 2009;15(4):283-93.

32. Yu H, Pardoll D, Jove R. STATs in cancer inflammation and immunity: a leading role for STAT3. Nat Rev Cancer. 2009:9(11):798-809.

33. Bartoli M, Platt D, Lemtalsi T, Gu X, Brooks SE, Marrero MB, Caldwell RB. VEGF differentially activates STAT3 in microvascular endothelial cells. FASEB J. 2003;17(11):1562-4.

34. Leong H, Mathur PS, Greene GL. Green tea catechins inhibit angiogenesis through suppression of STAT3 activation. Breast Cancer Res Treat. 2009; 117(3):505-15.

35. Dong Y, Lu B, Zhang X, Zhang J, Lai L, Li D, Wu Y, Song Y, Luo J, Pang X, et al. Cucurbitacin $\mathrm{E}$, a tetracyclic triterpenes compound from Chinese medicine, inhibits tumor angiogenesis through VEGFR2-mediated Jak2STAT3 signaling pathway. Carcinogenesis. 2010;31(12):2097-104

36. Zhuang G, Wu X, Jiang Z, Kasman I, Yao J, Guan Y, Oeh J, Modrusan Z, Bais C, Sampath D, et al. Tumour-secreted miR-9 promotes endothelial cell migration and angiogenesis by activating the JAK-STAT pathway. EMBO J. 2012;31(17):3513-23

37. Umezu T, Tadokoro H, Azuma K, Yoshizawa S, Ohyashiki K, Ohyashiki JH. Exosomal miR-135b shed from hypoxic multiple myeloma cells enhances angiogenesis by targeting factor-inhibiting HIF-1. Blood. 2014;124(25):3748-57.

38. Di Modica M, Regondi V, Sandri M, lorio MV, Zanetti A, Tagliabue E, Casalini P, Triulzi T. Breast cancer-secreted miR-939 downregulates VE-cadherin and destroys the barrier function of endothelial monolayers. Cancer Lett. 2017; 384:94-100.

39. Zhou W, Fong MY, Min Y, Somlo G, Liu L, Palomares MR, Yu Y, Chow A, O'Connor ST, Chin AR, et al. Cancer-secreted miR-105 destroys vascular endothelial barriers to promote metastasis. Cancer Cell. 2014;25(4):501-15.

40. Singh R, Pochampally R, Watabe K, Lu Z, Mo YY. Exosome-mediated transfer of miR-10b promotes cell invasion in breast cancer. Mol Cancer. 2014;13:256.

41. Arshad F, Wang L, Sy C, Avraham S, Avraham HK. Blood-brain barrier integrity and breast cancer metastasis to the brain. Patholog Res Int. 2010; 2011:920509.

42. Lee TH, Avraham HK, Jiang S, Avraham S. Vascular endothelial growth factor modulates the transendothelial migration of MDA-MB-231 breast cancer cells through regulation of brain microvascular endothelial cell permeability. J Biol Chem. 2003;278(7):5277-84

43. Tominaga N, Kosaka N, Ono M, Katsuda T, Yoshioka Y, Tamura K, Lotvall J, Nakagama H, Ochiya T. Brain metastatic cancer cells release microRNA-181Ccontaining extracellular vesicles capable of destructing blood-brain barrier. Nat Commun. 2015;6:6716.

44. Hanahan D, Weinberg RA. Hallmarks of cancer: the next generation. Cell. 2011;144(5):646-74.

45. Tsai JH, Yang J. Epithelial-mesenchymal plasticity in carcinoma metastasis. Genes Dev. 2013;27(20):2192-206.
46. Park SM, Gaur AB, Lengyel E, Peter ME. The miR-200 family determines the epithelial phenotype of cancer cells by targeting the E-cadherin repressors ZEB1 and ZEB2. Genes Dev. 2008;22(7):894-907.

47. Le MT, Hamar P, Guo C, Basar E, Perdigao-Henriques R, Balaj L, Lieberman J. miR-200-containing extracellular vesicles promote breast cancer cell metastasis. J Clin Invest. 2014;124(12):5109-28.

48. Wu X, Somlo G, Yu Y, Palomares MR, Li AX, Zhou W, Chow A, Yen Y, Rossi $J$ J, Gao H, et al. De novo sequencing of circulating miRNAs identifies novel markers predicting clinical outcome of locally advanced breast cancer. J Transl Med. 2012;10:42

49. Fong MY, Zhou W, Liu L, Alontaga AY, Chandra M, Ashby J, Chow A, O'Connor ST, Li S, Chin AR, et al. Breast-cancer-secreted miR-122 reprograms glucose metabolism in premetastatic niche to promote metastasis. Nat Cell Biol. 2015;17(2):183-94.

50. Medzhitov R. Toll-like receptors and innate immunity. Nat Rev Immunol. 2001;1(2):135-45.

51. Lund JM, Alexopoulou L, Sato A, Karow M, Adams NC, Gale NW, Iwasaki A, Flavell RA. Recognition of single-stranded RNA viruses by Toll-like receptor 7. Proc Natl Acad Sci U S A. 2004;101(15):5598-603.

52. Heil F, Hemmi H, Hochrein $H$, Ampenberger F, Kirschning C, Akira S, Lipford $\mathrm{G}$, Wagner H, Bauer S. Species-specific recognition of single-stranded RNA via toll-like receptor 7 and 8. Science. 2004;303(5663):1526-9.

53. Fabbri M, Paone A, Calore F, Galli R, Gaudio E, Santhanam R, Lovat F, Fadda P, Mao C, Nuovo GJ, et al. MicroRNAs bind to Toll-like receptors to induce prometastatic inflammatory response. Proc Natl Acad Sci U S A. 2012; 109(31):E2110-6.

54. Zhou M, Chen J, Zhou L, Chen W, Ding G, Cao L. Pancreatic cancer derived exosomes regulate the expression of TLR4 in dendritic cells via miR-203. Cell Immunol. 2014;292(1-2):65-9.

55. Ding G, Zhou L, Qian Y, Fu M, Chen J, Chen J, Xiang J, Wu Z, Jiang G, Cao L. Pancreatic cancer-derived exosomes transfer miRNAs to dendritic cells and inhibit RFXAP expression via miR-212-3p. Oncotarget. 2015;6(30):29877-88.

56. Wei Y, Lai X, Yu S, Chen S, Ma Y, Zhang Y, Li H, Zhu X, Yao L, Zhang J. Exosomal miR-221/222 enhances tamoxifen resistance in recipient ERpositive breast cancer cells. Breast Cancer Res Treat. 2014;147(2):423-31.

57. Challagundla KB, Wise PM, Neviani $P$, Chava H, Murtadha M, Xu T, Kennedy $\mathrm{R}$, Ivan C, Zhang $X$, Vannini I et al. Exosome-mediated transfer of microRNAs within the tumor microenvironment and neuroblastoma resistance to chemotherapy. J Natl Cancer Inst 2015, 107(7).

58. Zitvogel L, Regnault A, Lozier A, Wolfers J, Flament C, Tenza D, RicciardiCastagnoli P, Raposo G, Amigorena S. Eradication of established murine tumors using a novel cell-free vaccine: dendritic cell-derived exosomes. Nat Med. 1998:4(5):594-600

59. Hanahan D, Coussens LM. Accessories to the crime: functions of cells recruited to the tumor microenvironment. Cancer Cell. 2012;21(3):309-22.

60. Johnston LA. Competitive interactions between cells: death, growth, and geography. Science. 2009;324(5935):1679-82.

61. Diaz B, Moreno E. The competitive nature of cells. Exp Cell Res. 2005; 306(2):317-22.

62. Kosaka N, Iguchi H, Yoshioka Y, Hagiwara K, Takeshita F, Ochiya T. Competitive interactions of cancer cells and normal cells via secretory microRNAs. J Biol Chem. 2012;287(2):1397-405.

63. Au Yeung $C L$, Co NN, Tsuruga $T$, Yeung TL, Kwan SY, Leung CS, Li Y, Lu ES, Kwan K, Wong KK, et al. Exosomal transfer of stroma-derived miR21 confers paclitaxel resistance in ovarian cancer cells through targeting APAF1. Nat Commun. 2016;7:11150

64. Spizzo R, Almeida MI, Colombatti A, Calin GA. Long non-coding RNAs and cancer: a new frontier of translational research? Oncogene. 2012;31(43):4577-87.

65. Mercer TR, Mattick JS. Structure and function of long noncoding RNAs in epigenetic regulation. Nat Struct Mol Biol. 2013;20(3):300-7.

66. Ahadi A, Khoury S, Losseva M, Tran N. A comparative analysis of IncRNAs in prostate cancer exosomes and their parental cell lines. Genom Data. 2016:9:7-9.

67. Takahashi K, Yan IK, Wood J, Haga H, Patel T. Involvement of extracellular vesicle long noncoding RNA (linc-VLDLR) in tumor cell responses to chemotherapy. Mol Cancer Res. 2014;12(10):1377-87.

68. Gezer U, Ozgur E, Cetinkaya M, Isin M, Dalay N. Long non-coding RNAs with low expression levels in cells are enriched in secreted exosomes. Cell Biol Int 2014:38(9):1076-9.

69. Xue D, Zhou C, Lu H, Xu R, Xu X, He X. LncRNA GAS5 inhibits proliferation and progression of prostate cancer by targeting miR-103 through AKT/ mTOR signaling pathway. Tumor Biol. 2016;37(12):16187-97. 
70. Schneider C, King RM, Philipson L. Genes specifically expressed at growth arrest of mammalian cells. Cell. 1988;54(6):787-93.

71. Huarte M, Guttman M, Feldser D, Garber M, Koziol MJ, Kenzelmann-Broz D, Khalil AM, Zuk O, Amit I, Rabani M, et al. A large intergenic noncoding RNA induced by p53 mediates global gene repression in the p53 response. Cell. 2010;142(3):409-19.

72. Kino T, Hurt DE, Ichijo T, Nader N, Chrousos GP. Noncoding RNA gas5 is a growth arrest- and starvation-associated repressor of the glucocorticoid receptor. Sci Signal. 2010;3(107):ra8.

73. Hewson C, Morris KV. Form and function of exosome-associated long noncoding RNAs in cancer. Curr Top Microbiol Immunol. 2016;394:41-56.

74. Berrondo C, Flax J, Kucherov V, Siebert A, Osinski T, Rosenberg A, Fucile C, Richheimer S, Beckham CJ. Expression of the long non-coding RNA HOTAIR correlates with disease progression in bladder cancer and is contained in bladder cancer patient urinary exosomes. PLoS One. 2016;11(1):e0147236.

75. Wang J, Zhou Y, Lu J, Sun Y, Xiao H, Liu M, Tian L. Combined detection of serum exosomal miR-21 and HOTAIR as diagnostic and prognostic biomarkers for laryngeal squamous cell carcinoma. Med Oncol. 2014;31(9):148.

76. Yang Z, Zhou L, Wu LM, Lai MC, Xie HY, Zhang F, Zheng SS. Overexpression of long non-coding RNA HOTAIR predicts tumor recurrence in hepatocellular carcinoma patients following liver transplantation. Ann Surg Oncol. 2011;18(5):1243-50.

77. Zhang ZZ, Shen ZY, Shen YY, Zhao EH, Wang M, Wang CJ, Cao H, Xu J. HOTAIR long noncoding RNA promotes gastric cancer metastasis through suppression of poly r(C)-binding protein (PCBP) 1. Mol Cancer Ther. 2015; 14(5):1162-70

78. Sun X, Du P, Yuan W, Du Z, Yu M, Yu X, Hu T. Long non-coding RNA HOTAIR regulates cyclin J via inhibition of microRNA-205 expression in bladder cancer. Cell Death Dis. 2015;6:e1907.

79. Sun R, Qin C, Jiang B, Fang S, Pan X, Peng L, Liu Z, Li W, Li Y, Li G. Downregulation of MALAT1 inhibits cervical cancer cell invasion and metastasis by inhibition of epithelial-mesenchymal transition. Mol Biosyst. 2016;12(3):952-62.

80. Yang L, Bai HS, Deng Y, Fan L. High MALAT1 expression predicts a poor prognosis of cervical cancer and promotes cancer cell growth and invasion. Eur Rev Med Pharmacol Sci. 2015;19(17):3187-93.

81. Emmrich S, Streltsov A, Schmidt F, Thangapandi VR, Reinhardt D, Klusmann $\mathrm{JH}$. LincRNAs MONC and MIR100HG act as oncogenes in acute megakaryoblastic leukemia. Mol Cancer. 2014;13:171.

82. Sukowati $\mathrm{CH}$, Anfuso B, Torre G, Francalanci $P$, Croce LS, Tiribelli C. The expression of CD90/Thy-1 in hepatocellular carcinoma: an in vivo and in vitro study. PLoS One. 2013;8(10):e76830.

83. Lingala S, Cui YY, Chen X, Ruebner BH, Qian XF, Zern MA, Wu J. Immunohistochemical staining of cancer stem cell markers in hepatocellular carcinoma. Exp Mol Pathol. 2010;89(1):27-35.

84. Yu XH, Xu LB, Liu C, Zhang R, Wang J. Clinicopathological characteristics of 20 cases of hepatocellular carcinoma with bile duct tumor thrombi. Dig Dis Sci. 2011;56(1):252-9.

85. Lu JW, Chang JG, Yeh KT, Chen RM, Tsai JJ, Hu RM. Overexpression of Thy1/ CD90 in human hepatocellular carcinoma is associated with HBV infection and poor prognosis. Acta Histochem. 2011;113(8):833-8.

86. Conigliaro A, Costa V, Lo Dico A, Saieva L, Buccheri S, Dieli F, Manno M, Raccosta S, Mancone C, Tripodi M, et al. CD90+ liver cancer cells modulate endothelial cell phenotype through the release of exosomes containing H19 IncRNA. Mol Cancer. 2015;14:155.

87. Takahashi K, Yan IK, Haga H, Patel T. Modulation of hypoxia-signaling pathways by extracellular linc-RoR. J Cell Sci. 2014;127(Pt 7):1585-94.

88. Loewer S, Cabili MN, Guttman M, Loh YH, Thomas K, Park IH, Garber M, Curran M, Onder T, Agarwal S, et al. Large intergenic non-coding RNA-RoR modulates reprogramming of human induced pluripotent stem cells. Nat Genet. 2010;42(12):1113-7.

89. Nath B, Szabo G. Hypoxia and hypoxia inducible factors: diverse roles in liver diseases. Hepatology. 2012;55(2):622-33.

90. Takahashi K, Yan IK, Kogure T, Haga H, Patel T. Extracellular vesicle-mediated transfer of long non-coding RNA ROR modulates chemosensitivity in human hepatocellular cancer. FEBS Open Bio. 2014;4:458-67.

91. Qu L, Ding J, Chen C, Wu ZJ, Liu B, Gao Y, Chen W, Liu F, Sun W, Li XF, et al. Exosome-transmitted IncARSR promotes sunitinib resistance in renal cancer by acting as a competing endogenous RNA. Cancer Cell. 2016;29(5):653-68.

92. Salmena L, Poliseno L, Tay $Y$, Kats L, Pandolfi PP. A ceRNA hypothesis: the Rosetta Stone of a hidden RNA language? Cell. 2011;146(3):353-8.
93. Tay $Y$, Rinn J, Pandolfi PP. The multilayered complexity of ceRNA crosstalk and competition. Nature. 2014;505(7483):344-52.

94. Qu S, Yang X, Li X, Wang J, Gao Y, Shang R, Sun W, Dou K, Li H. Circular RNA: a new star of noncoding RNAs. Cancer Lett. 2015;365(2):141-8.

95. Wang F, Nazarali AJ, Ji S. Circular RNAs as potential biomarkers for cancer diagnosis and therapy. Am J Cancer Res. 2016;6(6):1167-76.

96. Zheng Q, Bao C, Guo W, Li S, Chen J, Chen B, Luo Y, Lyu D, Li Y, Shi G, et al. Circular RNA profiling reveals an abundant circHIPK3 that regulates cell growth by sponging multiple miRNAs. Nat Commun. 2016;7:11215.

97. Li Y, Zheng Q, Bao C, Li S, Guo W, Zhao J, Chen D, Gu J, He X, Huang S. Circular RNA is enriched and stable in exosomes: a promising biomarker for cancer diagnosis. Cell Res. 2015;25(8):981-4.

98. Boyerinas B, Park SM, Hau A, Murmann AE, Peter ME. The role of let-7 in cell differentiation and cancer. Endocr Relat Cancer. 2010;17(1):F19-36.

99. Zhang L, Valencia CA, Dong B, Chen M, Guan PJ, Pan L. Transfer of microRNAs by extracellular membrane microvesicles: a nascent crosstalk model in tumor pathogenesis, especially tumor cell-microenvironment interactions. J Hematol Oncol. 2015;8:14.

100. Yang M, Chen J, Su F, Yu B, Su F, Lin L, Liu Y, Huang JD, Song E. Microvesicles secreted by macrophages shuttle invasion-potentiating microRNAs into breast cancer cells. Mol Cancer. 2011;10:117.

101. Umezu T, Ohyashiki K, Kuroda M, Ohyashiki JH. Leukemia cell to endothelial cell communication via exosomal miRNAs. Oncogene. 2013;32(22):2747-55.

102. Roccaro AM, Sacco A, Maiso P, Azab AK, Tai YT, Reagan M, Azab F, Flores LM,

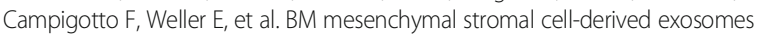
facilitate multiple myeloma progression. J Clin Invest. 2013;123(4):1542-55.

103. Alegre E, Sanmamed MF, Rodriguez C, Carranza O, Martin-Algarra S, Gonzalez A. Study of circulating microRNA-125b levels in serum exosomes in advanced melanoma. Arch Pathol Lab Med. 2014;138(6):828-32.

104. Kogure T, Lin WL, Yan IK, Braconi C, Patel T. Intercellular nanovesiclemediated microRNA transfer: a mechanism of environmental modulation of hepatocellular cancer cell growth. Hepatology. 2011;54(4):1237-48.

105. Corcoran C, Friel AM, Duffy MJ, Crown J, O'Driscoll L. Intracellular and extracellular microRNAs in breast cancer. Clin Chem. 2011;57(1):18-32.

106. Yuan JH, Yang F, Wang F, Ma JZ, Guo YJ, Tao QF, Liu F, Pan W, Wang TT, Zhou CC, et al. A long noncoding RNA activated by TGF-beta promotes the invasion-metastasis cascade in hepatocellular carcinoma. Cancer Cell. 2014; 25(5):666-81.

107. Wang F, Yuan JH, Wang SB, Yang F, Yuan SX, Ye C, Yang N, Zhou WP, Li WL, Li W, et al. Oncofetal long noncoding RNA PVT1 promotes proliferation and stem cell-like property of hepatocellular carcinoma cells by stabilizing NOP2. Hepatology. 2014;60(4):1278-90.

108. Salido-Guadarrama I, Romero-Cordoba S, Peralta-Zaragoza O, HidalgoMiranda A, Rodriguez-Dorantes M. MicroRNAs transported by exosomes in body fluids as mediators of intercellular communication in cancer. Onco Targets Ther. 2014;7:1327-38.

109. Lou G, Song X, Yang F, Wu S, Wang J, Chen Z, Liu Y. Exosomes derived from miR-122-modified adipose tissue-derived MSCs increase chemosensitivity of hepatocellular carcinoma. J Hematol Oncol. 2015;8:122.

110. Liu Y, Zhao L, Li D, Yin Y, Zhang CY, Li J, Zhang Y. Microvesicle-delivery miR150 promotes tumorigenesis by up-regulating VEGF, and the neutralization of miR-150 attenuate tumor development. Protein Cell. 2013:4(12):932-41.

111. Yim N, Ryu SW, Choi K, Lee KR, Lee S, Choi H, Kim J, Shaker MR, Sun W, Park $\mathrm{JH}$, et al. Exosome engineering for efficient intracellular delivery of soluble proteins using optically reversible protein-protein interaction module. Nat Commun. 2016;7:12277.

112. Zhang X, Yuan X, Shi H, Wu L, Qian H, Xu W. Exosomes in cancer: small particle, big player. J Hematol Oncol. 2015;8:83. 